\title{
Fosfato $\beta$-Tricálcico Monobloco no Tratamento de Fraturas Proximais do Úmero Cirúrgicas
}

\section{$\beta$-Tricalcium Phosphate in the Surgical Treatment of Proximal Humeral Fractures}

\author{
Marco SARMENTO $\rrbracket^{1,2}$, Samuel MARTINS ${ }^{1,2}$, Jacinto MONTEIRO ${ }^{1,2}$ \\ Acta Med Port 2016 Jan;29(1):41-45
}

RESUMO

Introdução: As fraturas proximais do úmero são cada vez mais frequentes, com maior tendência para o seu tratamento cirúrgico, predominado a utilização da osteossíntese com placa e parafusos bloqueados. Pela falência mecânica e biológica, apesar da evolução deste tipo de implantes, a utilização de enxertos ósseos sintéticos passaram a ser uma opção.

Material e Métodos: Num período de 96 meses, avaliámos os doentes com fraturas proximais do úmero, tratados cirurgicamente com placa e parafusos bloqueados e nos quais foi usado enxerto ósseo sintético de fosfato $\beta$-tricálcico. Avaliaram-se os resultados funcionais pelo arco de mobilidade e pelos exames radiográficos.

Resultados: Nos 19 doentes avaliados, para um follow-up médio de 53 meses, obteve-se um arco de mobilidade com valores médio de abdução de $140^{\circ}$, flexão anterior de $142^{\circ}$, rotação externa de $37^{\circ}$ e rotação interna com mão a L3, para um ângulo cefalo-diafisário de $136^{\circ}$.

Discussão: A utilização de enxerto ósseo sintético de fosfato $\beta$-tricálcico permite a estabilização da redução após fixação das fraturas proximais do úmero estabilizadas com placa e parafusos bloqueados. Esta redução que se traduz na manutenção do ângulo cefalodiafisário, permite a obtenção de bons resultados funcionais como o demonstra o arco de mobilidade nos vários planos.

Conclusão: $O$ enxerto ósseo sintético de fosfato $\beta$-tricálcico deverá ser encarado como uma terapêutica auxiliar na osteossíntese extramedular das fraturas proximais do úmero, principalmente naquelas com maior dificuldade de manutenção da redução pela maior cominução do calcar medial.

Palavras-chave: Beta-Fosfato Tricálcico; Fixação Interna de Fraturas; Fraturas do Ombro; Substitutos Ósseos.

\section{ABSTRACT}

Introduction: The proximal humeral fractures are becoming more frequent, with a greater tendency for its surgical treatment by osteosynthesis with plate and locked screws. The mechanical and biological failure in these fractures and devices, despite the evolution of this type of implants, highlighted the synthetic bone grafts became an option.

Material and Methods: Over a period of 96 months, patients considered were those with proximal humeral fractures treated surgically with a plate and locked screws, and in which $\beta$-tricalcium phosphate bone graft had been used. Functional results were evaluated by the shoulder range of motion as the radiological results.

Results: In 19 patients, with a medial follow up of 53 months, we obtained an average shoulder range of motion of $140^{\circ}$ in abduction, $142^{\circ}$ in forward flexion, $37^{\circ}$ in external rotation and L3 hand position in internal rotation for a cefalo-diaphyseal angle of $136^{\circ}$.

Discussion: The $\beta$-tricalcium phosphate synthetic bone graft allows the maintenance of reduction after fixation of proximal humeral fractures stabilized with plate and locked screws. This reduction which means the maintenance of cefalo-diaphyseal angle is in close relationship with functional results as shown by shoulder range of motion in all planes.

Conclusion: The $\beta$-tricalcium phosphate synthetic bone graft should be seen as an adjuvant therapy in extramedullary fixation of proximal humeral fractures, especially those with greater comminution of the medial calcar.

Keywords: Bone Substitutes Beta-Tricalcium Phosphate; Fracture Fixation, Internal; Shoulder Fractures.

\section{INTRODUÇÃO}

Ao aumento da esperança média de vida da população corresponde uma maior incidência de fraturas da extremidade proximal do úmero., ${ }^{1,2} \mathrm{O}$ tratamento cirúrgico é cada vez mais a opção escolhida cujos procedimentos vão desde as suturas transósseas até à substituição protésica. ${ }^{3-8}$ No entanto, a redução cruenta e a osteossíntese com placa e parafusos bloqueados é provavelmente o procedimento mais utilizado nos dias de hoje.

Embora tenha havido uma significativa evolução deste tipo de implantes, com maior adequação anatómica e mais baixo perfil, a perda da redução e subsequente pior resultado funcional, continua a ser uma realidade. . $^{9-11}$
Para ultrapassar esta falência, essencialmente pela falta de apoio medial ao nível do calcar, 12,13 foram adotadas várias medidas adicionais para a evitar, tais como o uso de placa adicional a contornar o calcar ${ }^{13}$ até ao uso de enxerto autólogo ou homólogo, ${ }^{9,14}$ vascularizado ou não, enxerto sintético ${ }^{15}$ ou cimento. ${ }^{16}$

$\mathrm{Na}$ presente série, os autores avaliam a sua experiência na utilização de enxerto ósseo sintético de fosfato $\beta$-tricálcico no tratamento cirúrgico de fraturas proximais do úmero associado a placa e parafusos bloqueados. São apresentados os resultados radiográficos e clinico-funcionais.

\footnotetext{
1. Serviço de Ortopedia. Centro Hospitalar Lisboa Norte. Lisboa. Portugal.

2. Departamento de Ortopedia. Faculdade de Medicina. Universidade de Lisboa. Lisboa. Portugal.

$\square$ Autor correspondente: Marco Sarmento. marco.sarmento@sapo.pt

Recebido: 24 de abril de 2015 - Aceite: 02 de agosto de 2015 | Copyright $\odot$ Ordem dos Médicos 2016
} 


\section{MATERIAL E MÉTODOS}

Foram avaliados todos os doentes com fraturas proximais do úmero, submetidos a osteossíntese com placa com parafusos bloqueados Philos $^{\circledR}$ (Depuy/Synthes) em que houve necessidade de aporte de enxerto ósseo sintético monobloco em cilindro de fosfato $\beta$-tricálcico Chronos $^{\circledR}$ (Fig. 1) (Depuy/Synthes) num período de 96 meses.

Consideraram-se os mecanismos causais da lesão e as lesões traumáticas concomitantes.

Todas as fraturas foram classificadas pelo sistema de Neer. ${ }^{17}$

A avaliação radiográfica final permitiu determinar o ângulo cefalo-diafisário em posição de antero-posterior, a presença de necrose e colapso da cabeça umeral, assim como a perfuração da cabeça umeral causada pelos parafusos. Pela radiografia determinou-se o comportamento biológico do enxerto sintético.

A avaliação funcional foi obtida pela medição dos arcos de mobilidade no plano coronal, sagital e axial.

A valorização da dor quantificou-se através da escala visual analógica (escala de 0 a 10).

Foi determinado, ao longo do seguimento, a presença de infeção, reação de corpo estranho ao material implantado e falência de material de osteossíntese, bem como sobre a necessidade de reintervenção cirúrgica e o motivo para tal.

O estudo foi realizado de acordo com a declaração de Helsínquia.

\section{RESULTADOS}

No período considerado (julho de 2005 a julho de 2013), 19 doentes com fraturas proximais do úmero foram submetidos a osteossíntese com placa e parafusos bloqueados e

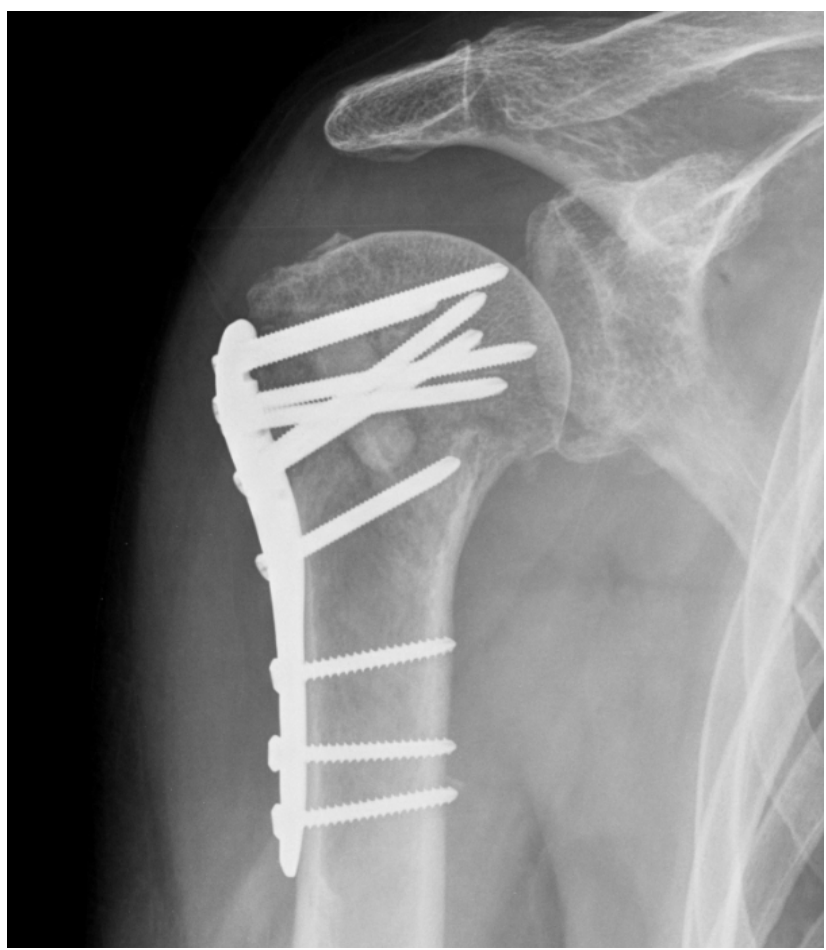

Figura 1 - Fratura com utilização de enxerto aporte de enxerto ósseo sintético de fosfato $\beta$-tricálcico.

Dez doentes eram do género feminino e nove do género masculino, com idade média de 60,1 anos (22-84 anos). A idade média para o género feminino foi de 70 anos (6084) e de 49,1 (22-72) anos para o género masculino.

O padrão de fratura mais frequente foi o de três partes, em 13 casos. Ocorreram quatro casos de fraturas Neer 4 , uma fratura-luxação em quatro partes e uma fratura Neer 2.

A grande maioria dos eventos traumáticos foram quedas simples de baixa energia (16 casos) e em três casos resultaram de traumatismos de alta energia no contexto de acidentes de viação (dois) e queda de altura (um). Todos os doentes apresentavam apenas queixas focais e monotraumáticas, com exceção de uma doente com traumatismo crânio-encefálico com hemorragia subaracnoideia, não submetida a qualquer intervenção por este motivo.

O período de follow-up médio foi de 53 meses, com um mínimo de 10 meses.

Na avaliação final obtivemos um ângulo cefalo-diafisário médio de $136^{\circ}$, com limites máximos de varismo de $110^{\circ}$ e de valgismo de $153^{\circ}$.

Não houve evolução para osteonecrose de nenhum dos casos, mas verificou-se a perfuração da cabeça umeral pelos parafusos bloqueados da cabeça em três doentes. Todos os enxertos sintéticos estavam osteointegrados.

Da avaliação funcional correspondente obtivemos uma avaliação analógica da dor média de 1 (0 - 4).

$\mathrm{O}$ arco de mobilidade médio da abdução foi de $140^{\circ}$ $\left(60^{\circ}-180^{\circ}\right)$, flexão anterior de $142^{\circ}\left(50^{\circ}-180^{\circ}\right)$, rotação externa, com o cotovelo apoiado ao tronco, de $37^{\circ}\left(0^{\circ}-90^{\circ}\right)$ e rotação interna com a mão a L3 (T7- sacro-ilíaca). (Tabela 1)

Quatro doentes foram submetidos a reintervenção cirúrgica do ombro em causa. Num doente foi feita descompressão subacromial artroscópica por conflito do troquiter após a sua medialização por perda da sua redução. Noutra doente fez-se também o mesmo procedimento e pelo mesmo motivo, mas por via aberta e associada à remoção do material de osteossíntese. Um doente desenvolveu um quadro de rigidez pós cirúrgica/traumática, com necessidade de capsulotomia gleno-umeral associada a remoção de material de osteossíntese. O quarto doente removeu o material de osteossíntese no âmbito de seguro de trabalho, sem nenhuma causa clínica.

Não se verificou nenhum caso de infeção nem de reação de corpo estranho correlacionadas com o material de implante ou com o enxerto ósseo.

\section{DISCUSSÃO}

A dificuldade no tratamento cirúrgico das fraturas proximais do úmero teve uma melhoria com o aparecimento das placas com parafusos bloqueados. No entanto, o seguimento destes doentes a médio/longo prazo veio demonstrar que as complicações das mesmas, embora em menor número, se mantiveram: o colapso da cabeça umeral associado à alteração do ângulo cefalo-diafisário (nomeadamente a varização do mesmo); a perfuração da cabeça 
Tabela 1 - Dados epidemiológicos e resultados obtidos

\begin{tabular}{|c|c|c|c|c|c|c|c|c|c|c|}
\hline Doente & Género & Lado & $\begin{array}{l}\text { Follow-up } \\
\text { (meses) }\end{array}$ & Tipo de \# & VAS & $\begin{array}{c}\text { Ângulo } \\
\text { cefalo- } \\
\text { diafisário } \\
\text { Final }\end{array}$ & Abdução & $\begin{array}{l}\text { Flexão } \\
\text { anterior }\end{array}$ & $\begin{array}{l}\text { Rotação } \\
\text { externa }\end{array}$ & $\begin{array}{c}\text { Rotação } \\
\text { interna }\end{array}$ \\
\hline 1 & $\mathrm{~F}$ & $\mathrm{D}$ & 24 & 3 & 4 & 142 & 120 & 120 & 40 & L3 \\
\hline 2 & $\mathrm{~F}$ & $E$ & 24 & 3 & 0 & 130 & 120 & 120 & 30 & L5 \\
\hline 3 & $M$ & $\mathrm{D}$ & 24 & 3 & 0 & 135 & 180 & 160 & 90 & $\mathrm{~T} 10$ \\
\hline 4 & M & $\mathrm{D}$ & 15 & 3 & 0 & 134 & 160 & 140 & 30 & $\mathrm{~T} 12$ \\
\hline 5 & $M$ & $\mathrm{D}$ & 15 & 4 & 0 & 110 & 100 & 100 & 15 & L3 \\
\hline 6 & $\mathrm{~F}$ & $E$ & 14 & 3 & 0 & 137 & 90 & 90 & 30 & L5 \\
\hline 7 & $\mathrm{~F}$ & D & 10 & 3 & 0 & 150 & 160 & 150 & 30 & $\mathrm{~T} 12$ \\
\hline 8 & $\mathrm{~F}$ & $\mathrm{D}$ & 10 & 3 & 1 & 146 & 180 & 150 & 30 & L5 \\
\hline 9 & M & $E$ & 61 & 4 & 1 & 132 & 150 & 170 & 45 & L1 \\
\hline 10 & $M$ & $E$ & 103 & 2 & 0 & 153 & 180 & 180 & 45 & $\mathrm{~T} 7$ \\
\hline 11 & M & $\mathrm{D}$ & 102 & $\begin{array}{c}4 \\
(\# L U X A C ̧ A ̃ O)\end{array}$ & 0 & 132 & 180 & 180 & 45 & L1 \\
\hline 12 & M & D & 101 & 4 & 3 & 145 & 60 & 150 & 30 & L1 \\
\hline 13 & $\mathrm{~F}$ & $\mathrm{D}$ & 75 & 4 & 3 & 120 & 60 & 60 & 15 & SI \\
\hline 14 & M & $E$ & 59 & 3 & 3 & 130 & 180 & 180 & 45 & $\mathrm{~T} 7$ \\
\hline 15 & $\mathrm{~F}$ & D & 60 & 3 & 3 & 124 & 180 & 180 & 45 & L1 \\
\hline 16 & $F$ & $E$ & 66 & 3 & 0 & 153 & 120 & 120 & 0 & L5 \\
\hline 17 & $\mathrm{~F}$ & $D$ & 107 & 3 & 3 & 133 & 170 & 170 & 45 & $\mathrm{~T} 7$ \\
\hline 18 & $F$ & $D$ & 65 & 3 & 2 & 135 & 120 & 140 & 30 & L1 \\
\hline 19 & $M$ & $E$ & 76 & 3 & 3 & 135 & 150 & 140 & 45 & T10 \\
\hline
\end{tabular}

umeral pelos parafusos proximais bloqueados; a necrose da cabeça umeral; a limitação do arco de mobilidade, principalmente acima do plano da omoplata, subsequente; e a possibilidade de conflito mecânico pelo material de implante.

Estas complicações, tal como já demonstradas nos trabalhos de Hertel et al, são devidas fundamentalmente a dois tipos de factores: estrutural, pela grande cominução do calcar correspondente interferência no eixo cefalo-diafisário; e biológico, resultante da inviabilidade circulatória da cabeça umeral.

Nas fraturas proximais do úmero em jovens, com vários fragmentos individualizados ou com maior cominução, é frequente, na tentativa da sua osteossíntese não se conseguir uma redução anatómica com a osteossíntese rígida com placa e parafusos bloqueadas, tanto pela falta de suporte estrutural da cabeça umeral, como pela cominução do calcar medial ou pela sua curta altura.

Por esse motivo ponderámos a utilização de enxerto ósseo sintético nas fraturas sempre que há maior cominução do calcar medial, maior dificuldade na manutenção da sua altura, ou dificuldade acrescida na manutenção da sua re- dução, por falta de estrutura óssea da cabeça umeral.

Introduzimos esse enxerto sintético de fosfato $\beta$-tricálcico na forma de cilindro de $10 \mathrm{~mm}$ de altura, por janela óssea resultante da fratura, geralmente utilizando o traço de fratura existente entre o troquiter (grande tuberosidade) e o troquino (pequena tuberosidade) após a redução e antes da fixação, com alojamento do mesmo na região perto do calcar medial, com o objetivo de manter a altura do calcar medial e secundariamente o eixo cefalo-diafisário. (Fig. 2)

Nos 19 doentes em que utilizámos este enxerto, obtivemos uma manutenção da redução, para um follow-up médio acima dos quatro anos, com um ângulo cefalo-diafisário de $136^{\circ}$, similar ao eixo médio desse ângulo.

Graças à restituição desse eixo após a fratura proximal do úmero, foi possível obter um arco de mobilidade bom com flexão anterior e abdução média de $140^{\circ}$, com rotação externa de $35^{\circ}$ e rotação interna com mão a L3.

Nos doentes cuja consolidação ocorreu em varo (< $130^{\circ}$ ), quer por incapacidade técnica de manter o mesmo pela grande comunição do calcar, quer pela sua perda de altura, obtivemos os piores resultados funcionais. Nos dois 


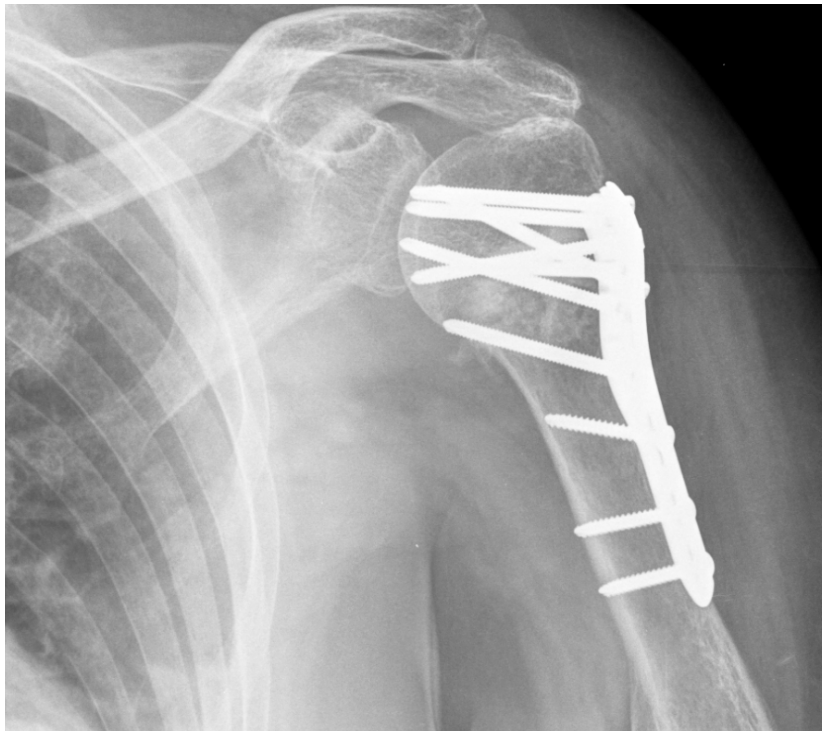

Figura 2 - Fratura consolidada com enxerto osteointegrado

doentes considerados, com ângulos cefalo-diafisários de $110^{\circ}$ e $120^{\circ}$, obtivemos amplitudes de abdução de $100^{\circ} \mathrm{e}$ $60^{\circ}$, flexão anterior de $100^{\circ}$ e $60^{\circ}$, rotação externa de $15^{\circ} \mathrm{e}$ rotação interna com mão a $L 3$ e sacro-ilíaca, respetivamente.

A perfuração da cabeça pelos parafusos bloqueados também foi simultânea com a consolidação em varo das cabeças umerais nos mesmos dois doentes. O mesmo se verificou no único doente cuja consolidação se verificou em valgo $\left(>150^{\circ}\right)$.

Um dos problemas possíveis na utilização de enxerto sintético é a sua não integração, maior probabilidade de reações tipo corpo estranho e infeçcão, mas que não ocorreram nesta nossa série. (Fig. 3)

Concomitantemente, a sua utilização pareceu favorecer a manutenção da viabilidade vascular da região cefálica, uma vez que não obtivemos nenhum caso de evolução para necrose da mesma.

Continuamos no entanto a verificar uma taxa de reintervenção cirúrgica neste tipo de patologia, mesmo com o uso de enxerto a rondar os $20 \%$. As causas continuam a ser as habituais, com maior predominância do conflito subacromial, provocado tanto pela perda de redução das tuberosidades como pelo conflito mecânico do material de implante. A rigidez articular surge com segunda causa, correlacionada com o traumatismo inicial ou com a agressão cirúrgica. Não nos parece haver, contudo, qualquer correlação direta com a utilização do enxerto sintético.

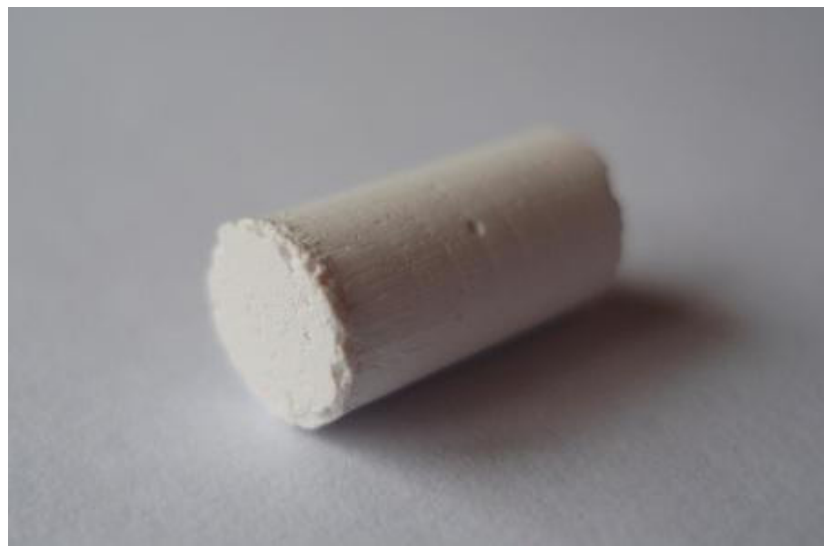

Figura 3 - Enxerto sintético fosfato beta-tricálcico

\section{CONCLUSÃO}

A utilização de enxerto ósseo sintético de fosfato tricálcico aparece como uma terapêutica auxiliar na osteossíntese das fraturas proximais do úmero. Parece-nos de maior utilidade nas fraturas com maior envolvimento cominutivo do calcar medial, em que existe dificuldade na manutenção da sua altura tanto na redução como após a osteossíntese.

Na nossa série, a sua correta utilização permitiu obter boas reduções das fraturas, sem colapso da cabeça, que se traduziram na manutenção do ângulo cefalo-diafisário proximal do úmero. Secundariamente, traduziram-se por arcos de mobilidade, nos vários planos, bons.

Consideramos que a sua introdução deve ser sistemática constituindo uma mais-valia sempre que houver dificuldade na redução e fixação por maior envolvimento do calcar medial.

\section{PROTECÇÃO DE PESSOAS E ANIMAIS}

Os autores declaram que os procedimentos seguidos estavam de acordo com os regulamentos estabelecidos pelos responsáveis da Comissão de Investigação Clínica e Ética e de acordo com a Declaração de Helsínquia da Associação Médica Mundial.

\section{CONFIDENCIALIDADE DOS DADOS}

Os autores declaram ter seguido os protocolos do seu centro de trabalho acerca da publicação dos dados de doentes.

\section{CONFLITOS DE INTERESSE}

Os autores declaram a inexistência de conflitos de interesse relativamente ao presente artigo.

\section{FONTES DE FINANCIAMENTO}

Não existiram fontes externas de financiamento para a realização deste artigo.

3. Resch H, Povacz P, Frohlich R, Wambacher M. Percutaneous fixation of three- and four-part fractures of the proximal humerus. J Bone Joint Surg Br. 1997;79:295-300.

4. Lin CL, Hong CK, Jou IM, Lin CJ, Su FC, Su WR. Suture anchor versus screw fixation for greater tuberosity fractures of the humerus - a 
biomechanical study. J Orthop Res. 2012;30:423-8.

5. Zhu Y, Lu Y, Shen J, Zhang J, Jiang C. Locking intramedullary nails and locking plates in the treatment of two-part proximal humeral surgical neck fractures: a prospective randomized trial with a minimum of three years of follow-up. J Bone Joint Surg Am. 2011;93:159-68.

6. Bastian JD, Hertel R. Osteosynthesis and hemiarthroplasty of fractures of the proximal humerus: outcomes in a consecutive case series. J Shoulder Elbow Surg. 2009;18:216-9.

7. Klein M, Juschka M, Hinkenjann B, Scherger B, Ostermann PA Treatment of comminuted fractures of the proximal humerus in elderly patients with the Delta III reverse shoulder prosthesis. J Orthop Trauma. 2008;22:698-704.

8. Levy J, Frankle M, Mighell M, Pupello D. The use of the reverse shoulder prosthesis for the treatment of failed hemiarthroplasty for proximal humeral fracture. J Bone Joint Surg Am. 2007;89:292-300.

9. Gardner MJ, Boraiah S, Helfet DL, Lorich DG. Indirect medial reduction and strut support of proximal humerus fractures using an endosteal implant. J Orthop Trauma. 2008;22:195-200.

10. Brunner F, Sommer C, Bahrs C, Heuwinkel R, Hafner C, Rillmann P, et al. Open reduction and internal fixation of proximal humerus fractures using a proximal humeral locked plate: a prospective multicenter analysis. J Orthop Trauma. 2009;23:163-72.
11. Sproul RC, lyengar JJ, Devcic Z, Feeley BT. A systematic review of locking plate fixation of proximal humerus fractures. Injury. 2011;42:408-13.

12. Gardner MJ, Weil Y, Barker JU, Kelly BT, Helfet DL, Lorich DG. The importance of medial support in locked plating of proximal humerus fractures. J Orthop Trauma. 2007;21:185-91.

13. Hertel R. Fractures of the proximal humerus in osteoporotic bone. Osteoporosis Int. 2005;16:S65-72.

14. Kim SH, Lee YH, Chung SW, Shin SH, Jang WY, Gong HS, et al. Outcomes for four-part proximal humerus fractures treated with a locking compression plate and an autologous iliac bone impaction graft. Injury. 2012;43:1724-31.

15. Somasundaram K, Huber CP, Babu V, Zadeh H. Proximal humeral fractures: the role of calcium sulphate augmentation and extended deltoid splitting approach in internal fixation using locking plates. Injury. 2013;44:481-7.

16. Unger S, Erhart S, Kralinger F, Blauth M, Schmoelz W. The effect of in situ augmentation on implant anchorage in proximal humeral head fractures. Injury. 2012;43:1759-63.

17. Neer CS 2nd. Displaced proximal humeral fractures. Part I. Classification and evaluation. Clin Orthop Relat Res. 1987;223:3-10. 